\begin{tabular}{|c|l|}
\hline Title & Identification of pulvomycin as an inhibitor of the futal osine pathway \\
\hline Author(s) & Ogasawara, Y asushi; U metsu, Shuhei; Inahashi, Y uki; Nonaka, Kenichi; Dairi, Tohru \\
\hline Citation & $\begin{array}{l}\text { Journal of antibiotics, 74, 825-829 } \\
\text { https://doi.org/10.1038/\$41429-021-00465-8 }\end{array}$ \\
\hline Issue Date & 2021-08-20 \\
\hline Doc URL & http://hdl.handle.net/2115/84174 \\
\hline Type & article (author version) \\
\hline File Information & manuscript-pulvomycin for HUSCAP.pdf \\
\hline
\end{tabular}

Instructions for use 


\title{
Identification of pulvomycin as an inhibitor of the futalosine pathway
}

Yasushi Ogasawara $^{1 *}$, Shuhei Umetsu ${ }^{2}$, Yuki Inahashi ${ }^{3}$, Kenichi Nonaka $^{3}$ and Tohru Dairi ${ }^{1 *}$

1) Graduate School of Engineering, Hokkaido University, Sapporo, Hokkaido 060-8628, Japan

2) Graduate School of Chemical Sciences and Engineering, Hokkaido University, Sapporo, Hokkaido 060-8628, Japan

3) Ōmura Satoshi Memorial Institute, Kitasato University, Minato-ku, Tokyo 108-8641, Japan

*Corresponding authors. E-mail: yogasawa@eng.hokudai.ac.jp and dairi@eng.hokudai.ac.jp

Keywords: futalosine pathway / inhibitor / menaquinone / pulvomycin

\begin{abstract}
Menaquinone is an essential cofactor in the electron-transfer pathway for bacteria. Menaquinone is biosynthesized from chorismate using either the well-known canonical pathway established by pioneering studies in model microorganisms or the futalosine pathway, which we discovered in Streptomyces. Because Helicobacter pylori, which causes stomach cancer, uses the futalosine pathway and most beneficial intestinal bacteria including lactobacilli use the canonical pathway, the futalosine pathway will be a great target to develop antibiotics specific for $H$. pylori. Here, we searched for such compounds from metabolites produced by actinomycetes and identified pulvomycin from culture broth of Streptomyces sp. K18-0194 as a specific inhibitor of the futalosine pathway.
\end{abstract}


Menaquinone (MK) is an indispensable lipid-soluble electron carrier in the respiratory chain for most bacteria. Two completely different MK biosynthetic pathways have been discovered. One is the well-known canonical pathway identified in model microorganisms such as Escherichia coli and Bacillus subtilis (Figure 1a). This pathway involves MenH-Menl, which biosynthesize MK from chorismate via o-succinylbenzoate as an intermediate.(ref. 1, 2) The other is the futalosine pathway that we previously identified in Streptomyces coelicolor (Figure 1b).(ref. 3-7) Analysis of publicly available genome database revealed that Helicobacter pylori, which causes stomach cancer, uses the futalosine pathway, while most beneficial intestinal bacteria including lactobacilli employ the canonical pathway. Thus, the futalosine pathway is an attractive target for the development of specific anti-H. pylori drugs. In this study, we searched for specific inhibitors targeting the futalosine pathway from metabolites produced by actinomycetes. 


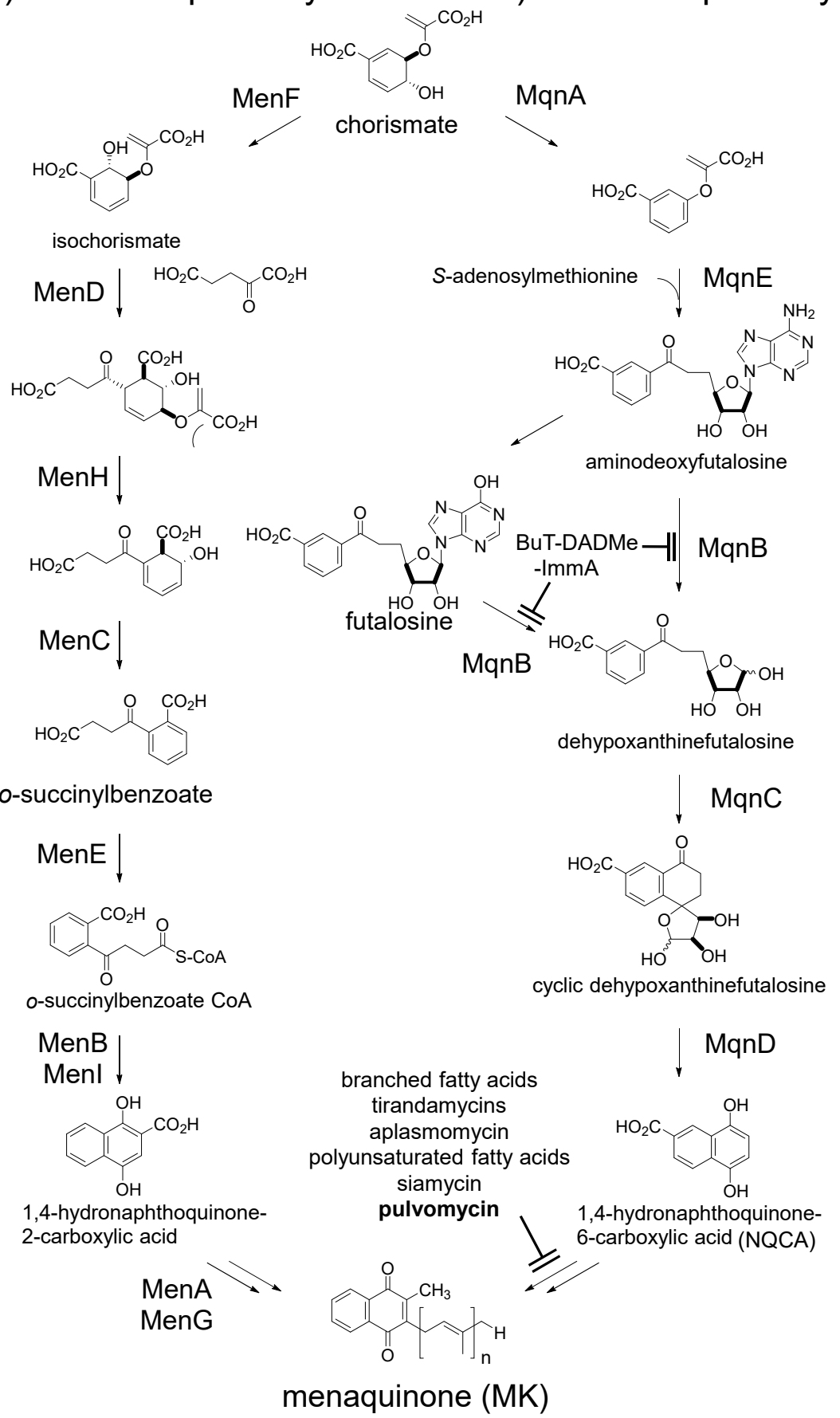

Figure 1. The two known menaquinone biosynthetic pathways. a) The canonical pathway and b) the futalosine pathway. In b), The targets of the known futalosine pathway inhibitors are shown. 
To identify samples containing futalosine pathway-specific inhibitors, the culture broths of approximately 1,000 actinomycetes were examined using a previously developed screening method.(ref. 8-10) In this method, two closely related Bacillus strains, Bacillus halodurans C-125 and Bacillus subtilis 168, were used as test organisms.(ref. 11) Although these strains exhibit a high degree of genome similarity, $B$. halodurans $C-125$ uses the futalosine pathway while $B$. subtilis 168 uses the canonical pathway. Considering that samples containing specific inhibitors would suppress the growth of $B$. halodurans $C-125$ but not of $B$. subtilis 168 , we selected such samples by paper disk assays and obtained 14 culture broths (1.4\%) as candidates. The candidates were further examined by a growth recovery assay with commercially available menaquinone- 4 (MK-4) supplementation. Consequently, we found that the growth inhibition of $B$. halodurans C125 by one sample (K18-0194) was recovered by supplementation of MK-4 $\left(0.1 \mathrm{mg} \mathrm{ml}^{-1}\right)$. We thus selected the culture broth of K18-0194 for further investigation. The strain K18-0194 was isolated from soil in Chiba Prefecture, Japan, and identified as Streptomyces based on the morphology and 16S rRNA gene sequence.

To isolate the active compound in the culture broth, we grew Streptomyces sp. K18-0194 in a $100-\mathrm{ml}$ test tube containing $10 \mathrm{ml} \mathrm{TSB}$ medium on a rotary shaker $(200 \mathrm{rpm})$ at $28^{\circ} \mathrm{C}$. After $48 \mathrm{~h}$, a $0.5 \mathrm{~mL}$ portion of the seed culture was transferred into a $200-\mathrm{ml}$ baffled Erlenmeyer flask containing $50 \mathrm{ml}$ of the production medium (oatmeal $1 \%$, pharmamedia $1 \%$, glucose $0.5 \%$, corn steep powder $0.5 \%, \mathrm{~K}_{2} \mathrm{HPO}_{4} 0.5 \%, \mathrm{MgSO}_{4} \cdot 7 \mathrm{H}_{2} \mathrm{O} 0.5 \%, \mathrm{FeSO}_{4} \cdot 7 \mathrm{H}_{2} \mathrm{O} 0.0001 \%, \mathrm{ZnSO}_{4} \cdot 7 \mathrm{H}_{2} \mathrm{O} 0.0001 \%$, $\left.\mathrm{CoCl}_{2} \cdot 6 \mathrm{H}_{2} \mathrm{O} 0.0001 \%, \mathrm{MnCl}_{2} \cdot 4 \mathrm{H}_{2} \mathrm{O} 0.0001 \%, \mathrm{CuSO}_{4} \cdot 5 \mathrm{H}_{2} \mathrm{O} 0.0001 \%, \mathrm{pH} 7.0\right)$ and cultivated on a rotary shaker $(200 \mathrm{rpm})$ at $30^{\circ} \mathrm{C}$ for $48 \mathrm{~h}$. Subsequently, the culture broth $(200 \mathrm{ml})$ was mixed with an equal volume of ethanol and the supernatant obtained by centrifugation was 
concentrated in vacuo. The residue was dissolved in water $(7 \mathrm{ml})$ and was subjected to reverse phase column chromatography using a Sep-Pak Vac 35cc C18 column (Waters) with step gradient elution ( $20 \%, 40 \%, 80 \%$, and $100 \%$ aq. acetonitrile). After concentration of the bioactive fractions eluted with $80 \%$ aq. acetonitrile, the resulting residue was further purified by HPLC (column: Kanto Mightysil Aqua RP-18 column $(250 \times 4.6 \mathrm{~mm})$, column temperature: $40^{\circ} \mathrm{C}$, mobile phase A: water, mobile phase B: acetonitrile, gradient: $0 \%$ solvent B for $0-5$ min and a linear gradient to $100 \%$ solvent $B$ for an additional $30 \mathrm{~min}$, flow rate: $1 \mathrm{ml} \mathrm{min}{ }^{-1}$, detection: photo diode array detector $190-400 \mathrm{~nm}$ ). By fractionation and bioassay, we identified the active compound eluted at a retention time of $28.6 \mathrm{~min}$ (Figure $2 \mathrm{~A}$ ). We collected the active compound by repetitive HPLC fractionation and obtained $0.7 \mathrm{mg}$ of compound (1) as a pale yellow solid. The high resolution ESI-MS and ${ }^{13} \mathrm{C}$ NMR spectra of 1 indicated that its molecular formula was $\mathrm{C}_{47} \mathrm{H}_{66} \mathrm{O}_{13}\left([\mathrm{M}+\mathrm{Na}]^{+}\right.$ calculated for $\mathrm{C}_{47} \mathrm{H}_{66} \mathrm{O}_{13} \mathrm{Na}^{+}$: 861.4396; found: 861.4391). Analysis of the 1D- and 2D-NMR $\left({ }^{1} \mathrm{H}\right.$ and ${ }^{13} \mathrm{C}, \mathrm{COSY}, \mathrm{HSQC}, \mathrm{HMBC}$ and ROESY) spectra of compound 1 (Figures S1-9 and Table S1) established the planar structure from C-1 through C-47, although 2D correlation between C-1 and C-21 was not observed. By comparing the spectral data with a previous report together with its characteristic UV-vis spectrum, compound $\mathbf{1}$ was determined to be pulvomycin (1, Figure 2B).(ref. 12) 

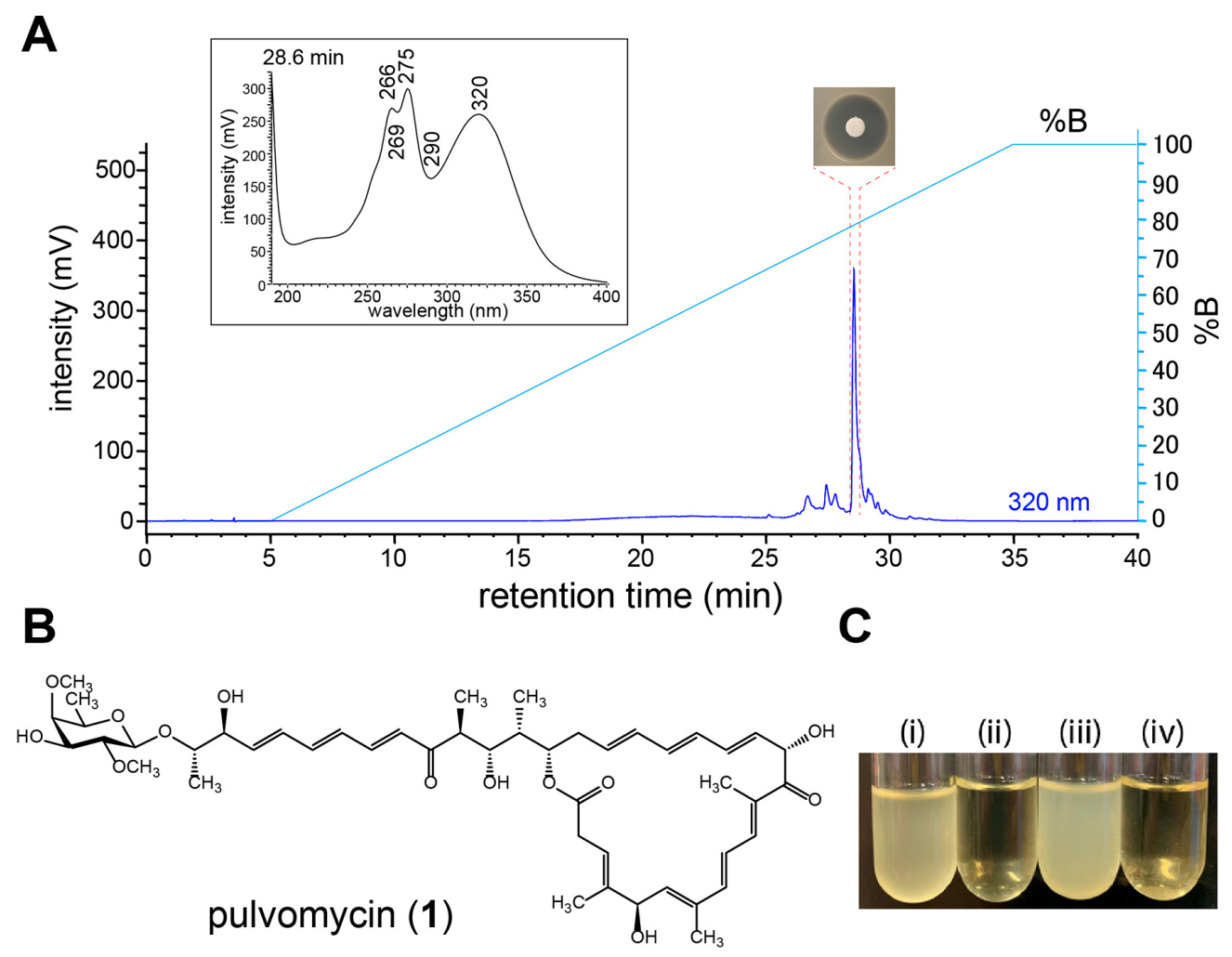

Figure 2. Isolation and characterization of the active compound. (A) Purification of 1 using HPLC by monitoring UV absorption at $320 \mathrm{~nm}$ (left scale) and the bioassay result against $B$. halodurans of the peak at retention time of 28.6 min. Gradient time program (\%B) was shown with line (right scale). The inlet shows UV spectrum of the peak. (B) Structure of pulvomycin (1). (C) Growth recovery assay of $B$. halodurans $C-125$. (i) $B$. halodurans only, (ii) with $1\left(0.2 \mu \mathrm{g} \mathrm{ml}^{-1}\right)$, (iii) with 1 $\left(0.2 \mu \mathrm{g} \mathrm{ml}^{-1}\right)$ and MK-4 $\left(100 \mu \mathrm{g} \mathrm{ml}^{-1}\right)$, and (iv) with $1\left(0.2 \mu \mathrm{g} \mathrm{ml}^{-1}\right)$ and NQCA $\left(20 \mu \mathrm{g} \mathrm{ml}^{-1}\right)$.

Pulvomycin (1) is a polyketide antibiotic that was originally isolated from an unidentified Streptomyces strain in 1957 and rediscovered in 1963 as labilomycin from Streptomyces albosporeus var. labilomyceticus.(ref. 13, 14, 15) As the name labilomycin implies, pulvomycin is 
labile to light and heat and its structure was not determined until 1984.(ref. 12) Pulvomycin acts as a potent inhibitor of elongation factor Tu (EF-Tu) in protein synthesis by preventing the formation of the ternary complex between aminoacyl-tRNA and EF-Tu.GTP.(ref. 16-19) Pulvomycin shows antimicrobial activity against Gram-positive bacteria and its minimum inhibitory concentrations (MICs) for Micrococcus luteus $\left(1 \mu \mathrm{g} \mathrm{ml}^{-1}\right)$, Staphylococcus aureus (2-4

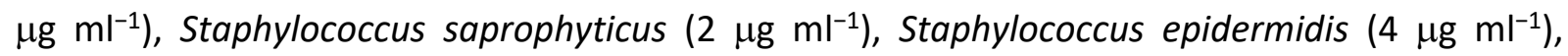
Enterococcus faecium $\left(4 \mu \mathrm{g} \mathrm{ml}^{-1}\right)$, and Enterococcus faecalis $\left(4-32 \mu \mathrm{g} \mathrm{ml}^{-1}\right)$, all of which use the canonical pathway for MK biosynthesis, have been reported.(ref. 20) By measuring the $\mathrm{OD}_{600}$ of liquid cultures containing various concentrations of $\mathbf{1}$, we determined the MIC value of 1 against B. halodurans C-125 was $200 \mathrm{ng} \mathrm{ml}^{-1}$, while its MIC value against Bacillus subtilis 168 was $10 \mu \mathrm{g}$ $\mathrm{ml}^{-1}$. We also confirmed that the growth inhibition of $B$. halodurans C-125 by compound $\mathbf{1}$ (200 $\mathrm{ng} \mathrm{ml}^{-1}$ ) was recovered by adding $0.1 \mathrm{mg} \mathrm{ml}^{-1} \mathrm{MK}-4$ (Figure $2 \mathrm{C}$ ). These results indicated that 1 inhibited both the futalosine pathway and protein synthesis with higher affinity toward enzymes in the futalosine pathway.

We next investigated the inhibition target step of 1 in the futalosine pathway by growth recovery experiments. Because the intermediate 1,4-hydronaphthoquinone-6-carboxylic acid (NQCA) was readily available and we previously showed that NQCA is membrane-permeable by growth recovery experiments with Streptomyces coelicolor mutants disrupted at SCO4506 (mqnA) and SCO4550 (mqnC), the genes responsible for the biosynthetic steps before NQCA formation, we examined whether the growth of $B$. halodurans $C-125$ was recovered with NQCA supplementation.(ref. 4) As shown in Figure 2C, inhibition of B. halodurans C-125 by 1 (200 ng 
$\mathrm{ml}^{-1}$ ) was not recovered with supplementation of NQCA $\left(0.02 \mathrm{mg} \mathrm{ml}^{-1}\right)$. Thus, compound $\mathbf{1}$ is suggested to inhibit a biosynthetic step between NQCA and MK. Although the detailed biosynthetic process from NQCA to MK is not clear at this point, it requires three enzymes, a prenyltransferase, a methyltransferase, and a decarboxylase. Because 1 contains a methylbranched polyene moiety, 1 might inhibit the enzymes after the prenylation step by mimicking the isoprenyl side chain of a biosynthetic intermediate.

To date, branched fatty acids, tirandamycin, aplasmomycin, boromycin, polyunsaturated fatty acids, siamycin, and BuT-DADMe-ImmA have been identified as specific inhibitors of the futalosine pathway.(ref. 8-10, 21, 22) Among these, BuT-DADMe-ImmA was shown to inhibit 6amino-6-deoxyfutalosine $N$-ribosylhydrolase (MqnB) while the others inhibit the conversion step from NQCA to MK. Although structure-activity relationship studies on 1 will be necessary to develop more specific inhibitors, the present study adds the polyene polyketide pulvomycin as a lead compound among futalosine pathway-specific inhibitors.

The authors declare no conflicts of interest.

\section{Acknowledgements}

This work was supported by Grants-in-Aid for Scientific Research on Innovative Areas from MEXT, Japan (JSPS KAKENHI Grant Number JP16H06452 to T.D.), Grants-in-Aid for Scientific Research from JSPS (JP18H03937 to T.D. JP18K05449 to Y.O.), and the Toyota Riken Scholar Program to Y.O. We also thank Dr. Eri Fukushi at the GC-MS and NMR Laboratory, Graduate 
School of Agriculture, Hokkaido University for acquiring NMR spectra. We thank Robbie Lewis, MSc, from Edanz (https://jp.edanz.com/) for editing a draft of this manuscript.

Supplementary Information is available for this paper on The Journal of Antibiotics website. https://doi.org/10.1038/s41429-021-00465-8 


\section{References}

1. Bentley R, Meganathan R. Biosynthesis of vitamin K (menaquinone) in bacteria. Microbiol Rev. 1982; 46: 241-280.

2. Meganathan R. Biosynthesis of menaquinone (vitamin K2) and ubiquinone (coenzyme Q): a perspective on enzymatic mechanisms. Vitam Horm. 2001; 61: 173-218.

3. Seto H, et al. Studies on a new biosynthetic pathway for menaquinone. J Am Chem Soc. 2008; 130: $5614-5615$.

4. Hiratsuka T, et al. An alternative menaquinone biosynthetic pathway operating in microorganisms. Science. 2008; 321: 1670-1673.

5. Hiratsuka T, Itoh N, Seto H, Dairi T. Enzymatic properties of futalosine hydrolase, an enzyme essential to a newly identified menaquinone biosynthetic pathway. Biosci Biotechnol Biochem. 2009; 73: 1137-1141.

6. Arakawa C, et al. Diversity of the early step of the futalosine pathway. Antimicrob Agents Chemother. 2011; 55: 913-916.

7. Mahanta N, Fedoseyenko D, Dairi T, Begley TP. Menaquinone biosynthesis: formation of aminofutalosine requires a unique radical SAM enzyme. J Am Chem Soc. 2013; 135: 15318-15321.

8. Tanaka R, et al. Branched fatty acids inhibit the biosynthesis of menaquinone in Helicobacter pylori. J Antibiot. 2011; 64: 151-153.

9. Ogasawara Y, Kondo K, Ikeda A, Harada R, Dairi T. Identification of tirandamycins as specific inhibitors of the futalosine pathway. J Antibiot. 2017; 70: 798-800.

10. Shimizu $\mathrm{Y}$, Ogasawara $\mathrm{Y}$, Matsumoto A, Dairi T. Aplasmomycin and boromycin are specific inhibitors of the futalosine pathway. J Antibiot. 2018; 71: 968-970.

11. Takami H, Nakasone K, Takaki Y, Maeno G, Sasaki R, Masui N. Fuji F, Hirama C, Nakamura Y, Ogasawara N, Kuhara S, Horikoshi K. Complete genome sequence of the alkaliphilic bacterium 
Bacillus halodurans and genomic sequence comparison with Bacillus subtilis. Nucleic Acids Res. 2000, 28: 4317-4331.

12. Smith RJ, Williams H, Barna JCJ, McDermott IR, Haegele KD, Piriou F. Wagner J, Higgins W. Structure revision of the antibiotic pulvomycin. J Am Chem Soc. 1985, 107: 2849-2857.

13. Zief M, Woodside R, Schmitz H. Pulvomycin. Antibiot Chemothe. 1957, 7: 384-386.

14. Akita E, Maeda K, Umezawa H. Isolation and characterization of labilomycin, a new antibiotc. J Antibiot Ser A. 1963, 16: 147-151.

15. Schwartz JL, Tishler M, Arison BH, Shafer HM, Ōmura S. Identification of mycolutein and pulvomycin as aureothin and labilomycin respectively. J Antibiot Ser A. 1976, 16: 147-151.

16. Wolf H, Assmann D, Fischer E. Pulvomycin, an inhibitor of protein biosynthesis preventing ternary complex formation between elongation factor Tu, GTP, and aminoacyl-tRNA. Proc Natl Acad Sci USA. $1978,75:$ 5324-5328.

17. Schmid B, Anke T, Wolf H. Action of pulvomycin and kirromycin on eukaryotic cells. FEBS Lett. 1978, 96: 189-191.

18. Pingoud A, Block W, Urbanke C, Wolf H. The antibiotics kirromycin and pulvomycin bind to different sites on the elongation factor Tu from Escherichia coli. Eur J Biochem. 1982, 123: 261265.

19. Parmeggiani A, Krab IM, Okamura S, Nielsen RC, Nyborg J, Nissen P. Structural basis of the action of pulvomycin and GE2270 A on elongation factor Tu. Biochemistry. 2006, 45: 6846-6857.

20. McKenzie NL, Thaker M, Koteva K, Hughes DW, Wright GD, Nodwell JR. Induction of antimicrobial activities in heterologous streptomycetes using alleles of the Streptomyces coelicolor gene absA1. J Antibiot. 2010, 63: 177-182.

21. Yamamoto $\mathrm{T}$, et al. Narrow-spectrum inhibitors targeting an alternative menaquinone biosynthetic pathway of Helicobacter pylori. J Infect Chemother. 2016; 22: 587-592. 
22. Wang S, et al. A picomolar transition state analogue inhibitor of MTAN as a specific antibiotic for Helicobacter pylori. Biochemistry. 2012; 51: 6892-6894. 